\title{
Origin of large magnetoresistance in the topological nonsymmorphic semimetal $\mathrm{TaSe}_{3}$
}

\author{
G. Gatti ${ }^{1,2,3,{ }^{*}}$ D. Gosálbez-Martínez $\odot,{ }^{1,4}$ Q. S. Wu, ${ }^{1,4}$ J. Hu, ${ }^{1,5}$ S. N. Zhang, ${ }^{1,4}$ G. Autès, ${ }^{1,4}$ M. Puppin $\odot,{ }^{6,2}$ D. Bugini ${ }^{\circledR},{ }^{7,8}$ \\ H. Berger, ${ }^{1}$ L. Moreschini $\odot,{ }^{9,}{ }^{10}$ I. Vobornik, ${ }^{11}$ J. Fujii ${ }^{\circledR},{ }^{11}$ J.- P. Ansermet ${ }^{\circ},{ }^{1}$ O. V. Yazyev ()$^{1,4}$ and A. Crepaldi ${ }^{1,2,7}$ \\ ${ }^{1}$ Institute of Physics, Ecole Polytechnique Fédérale de Lausanne (EPFL), CH-1015 Lausanne, Switzerland \\ ${ }^{2}$ Lausanne Centre for Ultrafast Science (LACUS), Ecole Polytechnique Fédérale de Lausanne (EPFL), CH-1015 Lausanne, Switzerland \\ ${ }^{3}$ Department of Quantum Matter Physics, University of Geneva, 24 Quai Ernest-Ansermet, 1211 Geneva, Switzerland \\ ${ }^{4}$ National Centre for Computational Design and Discovery of Novel Materials MARVEL, Ecole Polytechnique Fédérale de Lausanne (EPFL), \\ CH-1015 Lausanne, Switzerland \\ ${ }^{5}$ Fert Beijing Institute, School of Integrated Circuit Science and Engineering, Beihang University, Beijing 100191, China \\ ${ }^{6}$ Laboratory of Ultrafast Spectroscopy, ISIC, Ecole Polytechnique Fédérale de Lausanne (EPFL), CH-1015 Lausanne, Switzerland \\ ${ }^{7}$ Dipartimento di Fisica, Politecnico di Milano, Piazza Leonardo da Vinci 32, Milan 20133, Italy \\ ${ }^{8}$ Center for Nano Science and Technology@ PoliMi, Istituto Italiano di Tecnologia, Via Giovanni Pascoli 70/3, Milan 20133, Italy \\ ${ }^{9}$ Department of Physics, University of California, Berkeley, California 94720, USA \\ ${ }^{10}$ Materials Science Division, Lawrence Berkeley National Laboratory, Berkeley, California 94720, USA \\ ${ }^{11}$ Istituto Officina dei Materiali (IOM)-CNR, TASC Laboratory, Area Science Park-Basovizza, Trieste I-34149, Italy
}

(Received 1 July 2021; accepted 16 September 2021; published 13 October 2021)

\begin{abstract}
$\mathrm{TaSe}_{3}$ is a layered van der Waals semimetal with several inverted band gaps throughout the entire Brillouin zone and nontrivial $Z_{2}$ topological indices, which place it at the boundary between a strong and a weak topological phase. Our transport experiments reveal a quadratic nonsaturating magnetoresistance (MR) with values reaching $104 \%$ at $1.8 \mathrm{~K}$ and $14 \mathrm{~T}$, whose origins have to be searched in the material's band structure. Here we combine angle-resolved photoelectron spectroscopy experiments, also with spin resolution, with $a b$ initio calculations based on density functional theory in order to draw a connection between the Fermi surface topology and the measured transport properties. Simulations based on the calculated Fermi surface clarify that electron-hole compensation plays an important role for the observed MR in the bulk material. At the surface, the position of Fermi level differs, and it can be controlled by alkali metal deposition which accounts not only for the energy shift of the bands but it slightly modifies the dispersion of the valence and conduction bands. We propose that the observed band-gap renormalization might offer a route for engineering the topological phase in $\mathrm{TaSe}_{3}$, alternative to strain.
\end{abstract}

DOI: 10.1103/PhysRevB.104.155122

\section{INTRODUCTION}

The experimental observation of an increase in the resistivity of a crystal under application of an external magnetic field is as old as quantum mechanics [1]. Magnetoresistance (MR) has taken a central role in modern technology with the discovery of giant MR in metallic heterostructures [2,3], which served as basis for magnetic memories until the breakthrough of tunneling MR [4]. Owing to its commercial relevance [5,6], large efforts have been dedicated to foster the research in this field and new discoveries have periodically aroused the interest of the community, recently the observation of nonsaturating extreme MR in $\mathrm{WTe}_{2}$ [7].

This finding greatly contributed to bring nonmagnetic semimetals under the spotlight, in parallel with the advent of topological semimetals [8], and since then the two fields have grown intertwined [9]. In the case of topological fermions with linearly dispersing bands, reduced backscattering has

*gianmarco.gatti@unige.ch been invoked to explain the observation of linear nonsaturating MR [10,11]. Such topological protection is irrelevant for its quadratic counterpart, which is observed nowadays in an increasing number of semimetals [12-20]. In a minimal twoband model, the quadratic field dependence is the signature of perfect electron-hole compensation [21-23], which was initially put forward as the origin of the effect in $\mathrm{WTe}_{2}[7,24]$. The difficulties of $a b$ initio calculations to correctly estimate the size of the band gap, combined with the large sensitivity of the compensation upon the position of the Fermi level $\left(E_{\mathrm{F}}\right)$ [25], are compelling reasons to experimentally investigate the Fermi surface topology. High-resolution ARPES studies partially questioned the perfect electron-hole compensation [26], not only in $\mathrm{WTe}_{2}$ but also in other semimetals $[16,27]$. As a result, additional effects must contribute to the nonsaturating MR, such as temperature- and field-dependent changes in the band structure [28] and in the charge mobilities [25], due to the spin polarization of the band structure [29].

In this article we report on the observation of a quadratic nonsaturating MR in $\mathrm{TaSe}_{3}$ with values reaching $10^{4} \%$ at $1.8 \mathrm{~K}$ and $14 \mathrm{~T}$. We relate its origin to the Fermi surface 
topology by combining angle-resolved photoelectron spectroscopy (ARPES) and ab initio calculations based on density functional theory. $\mathrm{TaSe}_{3}$ is found at the boundary between a strong (STI) and a weak topological insulating phase (WTI), depending on the number of inverted band gaps [30]. Despite being a semimetal and not an insulator, the conduction (CB) and valence band (VB) are well separated in energy over the entire Brillouin zone (BZ), and $Z_{2}$ topological indices can be defined [31]. The Fermi surface consists of electron and hole pockets, whose perfect compensation is an important ingredient for the observed MR. In fact, by exploiting recently developed computational methods we are capable to reproduce the quadratic behavior of the MR in the bulk $\mathrm{TaSe}_{3}$ [23]. We notice that the experimental position of the Fermi level differs at the surface, as measured by ARPES, and it can be further modified by evaporation of an alkali metal (K). Interestingly, this is not responsible for a rigid band shift, but it induces modifications in the band structure, and we expect that this band-gap engineering might be generalized to devices that could tune the material across its topological phase diagram by an electric field.

\section{METHODS}

\section{A. $\mathrm{TaSe}_{3}$ crystal properties and synthesis}

$\mathrm{TaSe}_{3}$ belongs to the family of quasi-one-dimensional (1D) trichalcogenides, which in the past have been thoroughly investigated as a case study for charge density wave (CDW) ordering $[32,33]$. Unlike the related compounds, $\mathrm{TaSe}_{3}$ does not undergo a CDW transition [34,35]. It remains metallic down to $T=1.5 \mathrm{~K}$, where it becomes superconducting [36]. The monoclinic crystal structure belongs to the nonsymmorphic space group $P 2_{1} / m$ (No. 11) [37]. It is composed of four prismatic chains running along the $b$ crystallographic direction and weakly bounded by van der Waals forces [Fig. 1(a)]. The side view of Fig. 1(b) shows that the (101) surface, indicated by red lines, is the natural cleavage plane, as confirmed by independent studies [38,39]. At the macroscopic level, the material's 1D character is reflected in its crystal morphology: single crystals are needlelike, oriented along the $b$ direction, as shown in Fig. 1(c). High quality single crystals of TaSe3 are grown by direct sublimation of high purity $\mathrm{Ta}$ and $\mathrm{Se}$, as described in detail elsewhere $[35,40]$.

\section{B. ARPES and magnetotransport experiments}

ARPES and spin-resolved ARPES (srARPES) experiments were carried out at the low-energy branch of the APE beamline, at the Elettra synchrotron. The photon energy was varied in the range $50-83 \mathrm{eV}$, and the light was horizontally polarized. According to the acquisition geometry, this does not enforce any strict selection rule on the parity of the probed states [41]. The samples were exfoliated in ultrahigh vacuum via the scotch-tape method at room temperature, to expose atomically clean and flat (101) surfaces. The measurements were performed at liquid nitrogen temperature, and the combined energy and momentum resolution of the spectrometer were equal to $20 \mathrm{meV}$ and $0.2^{\circ}$, respectively. Deposition of potassium $(\mathrm{K})$ was achieved in situ by a commercial dispenser

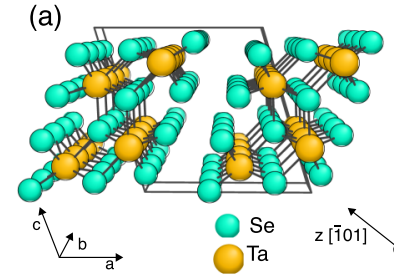

(d) (b)

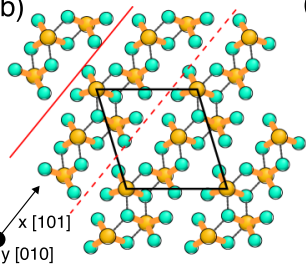

(e)
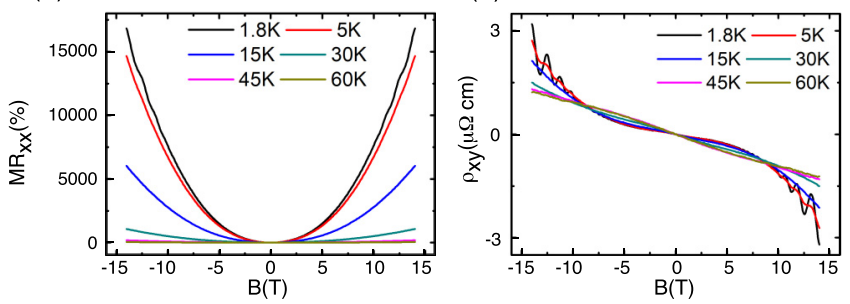

FIG. 1. (a) Crystal structure of $\mathrm{TaSe}_{3}$, made by prismatic chains of Ta (yellow) and Se (teal) atoms running along the $b$ crystallographic direction and weakly bounded by van der Waals interaction. (b) Side view of the crystal structure showing that the most favorable cleavage termination corresponds to the (101) plane. (c) Photo of $\mathrm{TaSe}_{3}$ single crystals several $\mathrm{cm}$ long along the $b$ direction. (d) Magnetoresistance (MR) as a percent variation of the longitudinal resistivity $\left(\rho_{x x}\right)$ with respect to the values at zero magnetic field. Each curve in (d) is normalized to the resistivity at the lowest temperature. At low temperature a quadratic nonsaturating MR develops. (e) Dependence of the Hall resistance $\rho_{x y}$ as a function of magnetic field, for various temperatures.

(SAES), properly outgassed in advance, and during evaporation the sample was kept at liquid nitrogen temperature.

For the spin-resolved (srARPES) experiments, the spin detection is based on high efficiency very low-energy electron diffraction (VLEED) on a $\mathrm{Fe}(001)-p(1 \times 1) \mathrm{O}$ target, whose magnetization axis and direction is controlled by coils [42]. The energy and angular resolution were $50 \mathrm{meV}$ and $0.75^{\circ}$, respectively. By exploiting the deflecting lens, the spin signal was acquired both along momentum distribution curves (MDCs) and energy distribution curves (EDCs). In both cases, from the measured majority $\left(I^{+}\right)$and minority $\left(I^{-}\right)$spin components, we have calculated the spin polarization defined as $P=\frac{\left(I^{+}-I^{-}\right)}{S\left(I^{+}+I^{-}\right)}$, where $S$ is the Sherman function. The value of $S=0.3$ was obtained by proper calibration on a $\mathrm{Au}(111)$ single crystal. Finally, the spin-up (spin-down) components are calculated following the relation $S_{\text {up/down }}=(1 \pm P)\left(I^{+}+I^{-}\right)$ as in Ref. [42].

The transport properties were probed by using a commercial Physical Property Measurement System (PPMS) from Quantum Design, operating in the temperature range 1.8$300 \mathrm{~K}$ with the possibility to apply a variable magnetic field in the range $\pm 14 \mathrm{~T}$. Samples were measured in a Hall bar geometry, with the magnetic field applied in the direction orthogonal to the (101) surface termination, while the electric field was applied parallel to the chain axis, along the $b$ crystallographic direction.

\section{First-principles calculations}

First-principles calculations were carried out with the experimental lattice parameters $(a=10.402 \AA, b=3.495 \AA$, 
$c=9.829 \AA$, and $\beta=106.26^{\circ}$ ) and the atomic positions from Ref. [40]. The calculations were performed within the density functional theory (DFT) formalism as implemented in the Quantum ESPRESSO package [43], using the generalized gradient approximation (GGA) and fully relativistic normconserving pseudopotential to account for spin-orbit effects. For the self-consistent calculations we used a $6 \times 16 \times 6 k$ mesh and an 80 Ry energy cutoff for the plane-wave expansion. The Fermi surface, and the momentum-resolved bulk and surface density of states using the semi-infinite Green's function technique [44], were obtained from the Wannier Hamiltonian using Wannier90 code [45].

Magnetoresistance was calculated using the combination of the Boltzmann transport theory and the Fermi surface obtained from first principles [23] which was implemented in an open-source software package WANNIERTOOLS [46]. This method is validated by many nonmagnetic metals and semimetals such as $\mathrm{Cu}$ [23], $\mathrm{Bi}$ [23], $\mathrm{WP}_{2}$ [23], $\mathrm{ZrSiS}$ [47], $\mathrm{MoO}_{2}$ [48], and $\mathrm{SiP}_{2}$ [49]. Within the relaxation time approximation, the band-wise conductivity tensor $\sigma$ is calculated by solving the Boltzmann equation in presence of an applied magnetic field as $[23,46,50,51]$

$$
\sigma^{(n)}(\mathbf{B})=\frac{e^{2}}{4 \pi^{3}} \int d \mathbf{k} \tau_{n} \mathbf{v}_{n}(\mathbf{k}) \overline{\mathbf{v}}_{n}(\mathbf{k})\left(-\frac{\partial f}{\partial \varepsilon}\right)_{\varepsilon=\varepsilon_{n}(\mathbf{k})},
$$

where $e$ is the electron charge, $n$ is the band index, $\tau_{n}$ is the relaxation time of $n$th band that is assumed to be independent on the wave vector $\mathbf{k}, f$ is the Fermi-Dirac distribution, $\mathbf{v}_{n}(\mathbf{k})$ is the group velocity defined by the gradient of band energy

$$
\mathbf{v}_{n}(\mathbf{k})=\frac{1}{\hbar} \nabla_{\mathbf{k}} \varepsilon_{n}(\mathbf{k})
$$

and $\overline{\mathbf{v}}_{n}(\mathbf{k})$ is the weighted average of velocity over the past history of the charge carrier

$$
\overline{\mathbf{v}}_{n}(\mathbf{k})=\int_{-\infty}^{0} \frac{d t}{\tau_{n}} e^{\frac{t}{\tau_{n}}} \mathbf{v}_{n}[\mathbf{k}(t)] .
$$

The orbital motion of charge carriers in applied magnetic field causes the time evolution of $\mathbf{k}_{n}(t)$, written as

$$
\frac{d \mathbf{k}_{n}(t)}{d t}=-\frac{e}{\hbar} \mathbf{v}_{n}[\mathbf{k}(t)] \times \mathbf{B},
$$

with $\mathbf{k}_{n}(0)=\mathbf{k}$. The total conductivity is the sum of bandwise conductivities, i.e., $\sigma=\sum_{n} \sigma^{(n)}$, which is then inverted to obtain the resistivity tensor $\rho=\sigma^{-1}$.

\section{RESULTS}

\section{A. Experimental magnetoresistance}

The measured MR is displayed in Fig. 1(d) as percent variation of the longitudinal resistivity $\left(\rho_{x x}\right)$ with respect to the values at zero magnetic field. In order to ease the comparison among the data set acquired at different temperatures, each curve in Fig. 1(d) is normalized to the resistivity at the lowest temperature. By decreasing the temperature, a quadratic nonsaturating MR develops, which gets as large as $1.5 \times 10^{4} \%$ at $1.8 \mathrm{~K}$ and for a $B$ field of $14 \mathrm{~T}$. At low temperatures we resolve quantum oscillations for large $B$ values, thus testifying the very high quality of our single crystals. The observation of quantum oscillations paves the way for investigating the transport properties of $\mathrm{TaSe}_{3}$ beyond the quantum limit. We leave these aspects for a future study, and we focus here on elucidating the origin of the MR effect. For completeness, Fig. 1(e) shows the measured Hall resistivity $\rho_{x y}$. At high temperature its behavior is well approximated by a line, whose slope denotes that electrons are the dominant charge carriers. By lowering the temperature, the change in $\rho_{x y}$ is nonmonotonic, in particular in the low field region the curves flatten and this is suggestive of a possible electron-hole compensation [23].

\section{B. Experimental and theoretical band dispersion}

In order to clarify the contribution of electron-hole compensation and other mechanisms to the measured MR, we have carried out a systematic investigation of the band structure of $\mathrm{TaSe}_{3}$ by combining ARPES with $a b$ initio calculations, whose results are summarized in Fig. 2. Figure 2(a) displays the bulk Brillouin zone (BZ), where the (101) projected surface BZ (SBZ) is indicated, as well (green rectangle). We indicate in yellow the plane orthogonal to the $b^{*}$ direction, with the aim to clarify the relative orientation of the $k_{z}$ axis, normal to the sample surface, with respect to the three-dimensional (3D) BZ. The $k_{z}$ direction, which is accessible to ARPES by performing photon energy dependent studies, forms an angle $\Theta \sim 3.5^{\circ}$ with respect to the $\Gamma D$ bulk direction. The dispersion along $k_{z}$ will be discussed in detail in Fig. 3. In Fig. 2(a) the high-symmetry points are indicated, as well, with the parity products of the valence band wave functions at the time-reversal-invariant momentum (TRIM) points. We anticipate that, according to our first-principle calculations, the material is in a weak topological phase described by $Z_{2}$ indices $(0 ; 1,0,1)$, but this will be discussed in depth later. The calculated bulk band structure of Fig. 2(b) clearly shows that $\mathrm{TaSe}_{3}$ is a semimetal, whose valence and conduction bands, highlighted in red and blue, are well separated in energy and both cross $E_{\mathrm{F}}$, thus contributing to the material's transport properties.

Figure 2(c) shows the photoemission intensity measured at $77 \mathrm{eV}$ photon energy along the $\bar{M}-\bar{X}-\bar{\Gamma}-\bar{Y}$ high-symmetry directions of the (101) SBZ. We resolve several bands; CB forms a shallow electronlike pocket with its minimum at a binding energy of $160 \pm 10 \mathrm{meV}$. The MDC integrated at $E_{\mathrm{F}}$ [Fig. 2(e)] shows that $\mathrm{CB}$ has Fermi wave vectors $k_{\mathrm{F} 2}=$ $0.09 \pm 0.01 \AA^{-1}$ and $k_{\mathrm{F} 3}=0.09 \pm 0.02 \AA^{-1}$, along $\overline{X M}$ and $\overline{\Gamma X}$ respectively.

The VB crosses $E_{\mathrm{F}}$ along $\overline{\Gamma Y}$ with Fermi wave vector $k_{\mathrm{F} 1}=$ $0.04 \pm 0.01 \AA^{-1}$; the dispersion of this state is linear with an exceptionally large band velocity of $5 \times 10^{5} \mathrm{~m} / \mathrm{s}$, comparable to those of $\mathrm{Cd}_{3} \mathrm{As}_{2}$ [52,53] and $\mathrm{ZrTe}_{5}$ [54,55]. The momentum-resolved surface density of states, of Fig. 2(d), calculated for a semi-infinite crystal, reproduces well the experimental results, with small differences. The first is the position of $E_{\mathrm{F}}$, which is experimentally found at larger energies. We propose that this partial $n$ doping is due to defects created at the surface by cleavage. The second difference concerns the dispersion of $\mathrm{CB}$. Theory predicts the band to produce two contours at $E_{\mathrm{F}}$, and this has consequences on the topology of the Fermi contour that we will comment on later. Furthermore, according to Fig. 2(d) theory predicts CB to 

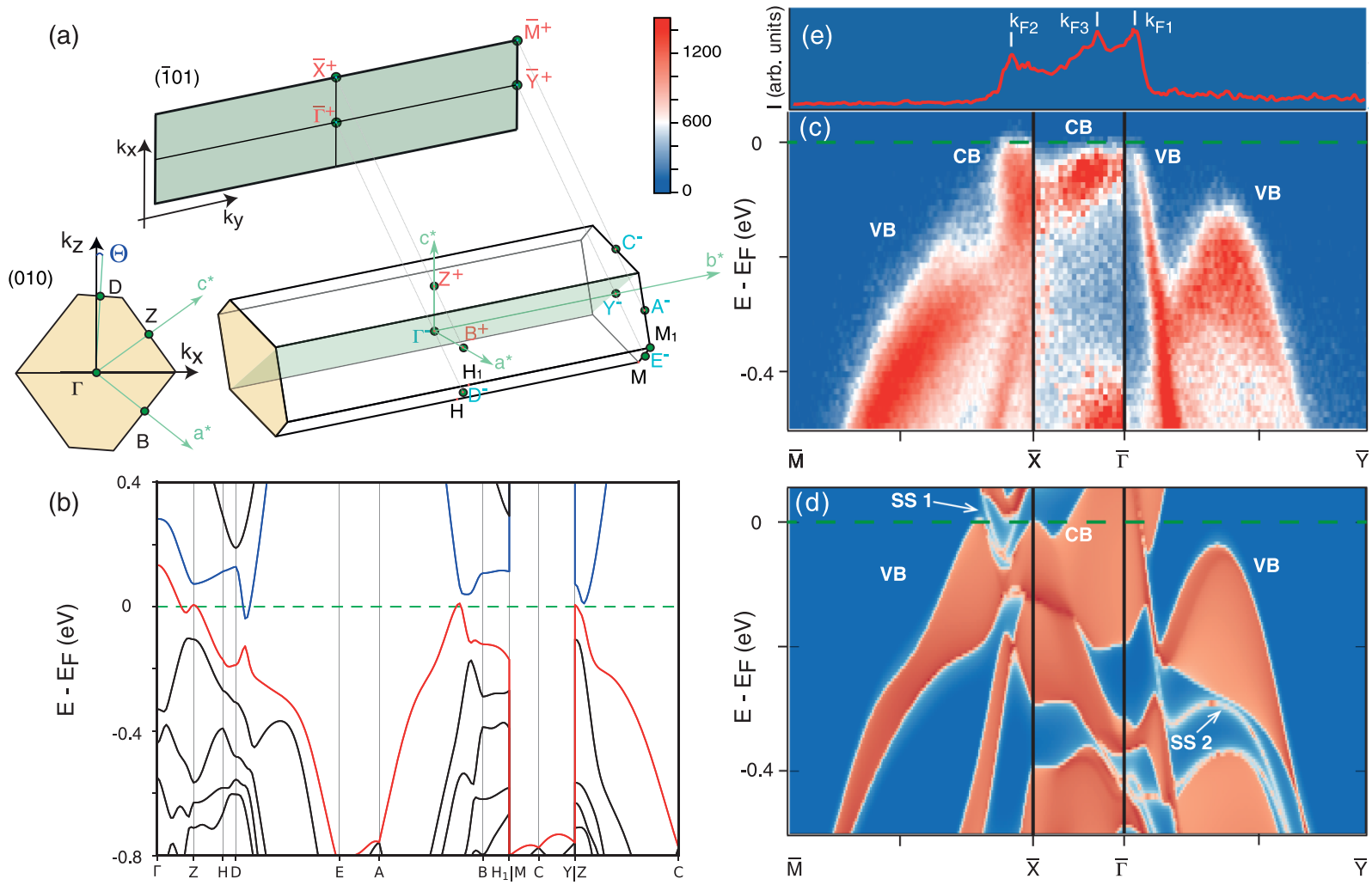

FIG. 2. (a) 3D bulk Brillouin zone (BZ). High-symmetry points are indicated along with the parity of the product of the valence band wave functions at the time-reversal-invariant momentum (TRIM) points. We illustrate also the BZ projected on the (101) surface termination, and the $2 \mathrm{D} \mathrm{BZ}$ orthogonal to the $b^{*}$ direction, which helps us in clarifying the relative orientation of the $k_{z}$ axis, orthogonal to the sample surface. (b) Calculated bulk band dispersion, red and blue lines trace the highest occupied VB and the lowest occupied CB. (c) Band dispersion measured at $77 \mathrm{eV}$ along the $\bar{M}-\bar{X}-\bar{\Gamma}-\bar{Y}$ high-symmetry directions of the (101) SBZ. Several states are resolved, corresponding to the conduction band (CB), the valence band (VB), and surface states (SS). (d) Momentum-resolved surface density of states calculated for a semi-infinite crystal along the same direction of (c). (e) Momentum distribution curve integrated from the data of (c) at $E_{\mathrm{F}}$ in order to highlight the position of the Fermi wave vectors $k_{\mathrm{F}}$.

disperse below $E_{\mathrm{F}}$ also along $\overline{\Gamma Y}$. This is due to the dispersion of the bulk state in the direction perpendicular to the sample surface. The calculations of Fig. 2(d) are integrated over all the possible $k_{z}$ values, while despite its well-known surface sensitivity, ARPES retains a partial $k_{z}$ resolution [56].

In order to better analyze the bulk dispersion of $\mathrm{CB}$ around the $\mathrm{BZ}$ center, we have varied the photon energy, and the results are summarized in Fig. 3. Figure 3(a) shows the dispersion along the $k_{z}$ direction, which corresponds approximately to the bulk $\Gamma D$ direction, as discussed previously in Fig. 2(a). We retrieve the $k_{z}$ values from the photon energy for a choice of the inner potential $V_{0}=10 \mathrm{eV}$, compatible with other ARPES study [39]. As a result, the data acquired at $77 \mathrm{eV}$ correspond to a curved surface in the reciprocal space passing near the $\Gamma$ point of the sixth BZ (red line). Whereas, for energy close to $65 \mathrm{eV}$, the ARPES data can be projected on a surface passing near $D$ (blue line). Figures 3(b) and 3(c) compare the band dispersion along $k_{y}$ and passing through those two points. While near $\Gamma$ [Fig. 3(c)], the linearly dispersing VB is well resolved, the extra intensity observed near $D$ [Fig. 3(b)] is attributed to dispersion of $\mathrm{CB}$ below $E_{\mathrm{F}}$ and the partial mixing of the VB and CB signals.

\section{Fermi surface and calculated magnetoresistance}

Having discussed the material's band dispersion over the 3D bulk BZ, we are now ready for analyzing the Fermi surface, with the aim to elucidate its topology and how it relates to the measured MR. Figures 3(d) and 3(f) show the measured Fermi surfaces for $\left(k_{x}, k_{y}\right)$ planes passing through $\Gamma$ and $D$, respectively. The dashed green rectangle delineates the boundary of the bulk BZ for the two $k_{z}$ values. In Fig. 3(d) we resolve two contours with ellipsoidal and rectangular shapes. We attribute the former to $\mathrm{CB}$, and the latter to $\mathrm{VB}$, on the basis of the evolution of the contours $75 \mathrm{meV}$ below $E_{\mathrm{F}}$ [Figs. 3(e) and 3(g)]. According to these contours, the area of $\mathrm{CB}$ is found to be $A_{\mathrm{CB}}=0.07 \pm 0.007 \AA^{-2}$, larger than the value estimated for $\mathrm{VB} A_{\mathrm{VB}}=0.014 \pm 0.007 \AA^{-2}$. Similarly, in Fig. 3(f) the area corresponding to the $\mathrm{CB}$ and VB contours are also different.

This apparent deviation from perfect electron-hole compensation is clarified by theory in Fig. 4. Figure 4(a) shows the 3D Fermi surface, where blue and red indicate electronlike and holelike states, respectively. We immediately notice that, despite the 1D character of the crystal, all the Fermi sheets form closed surfaces. In order to better compare to 


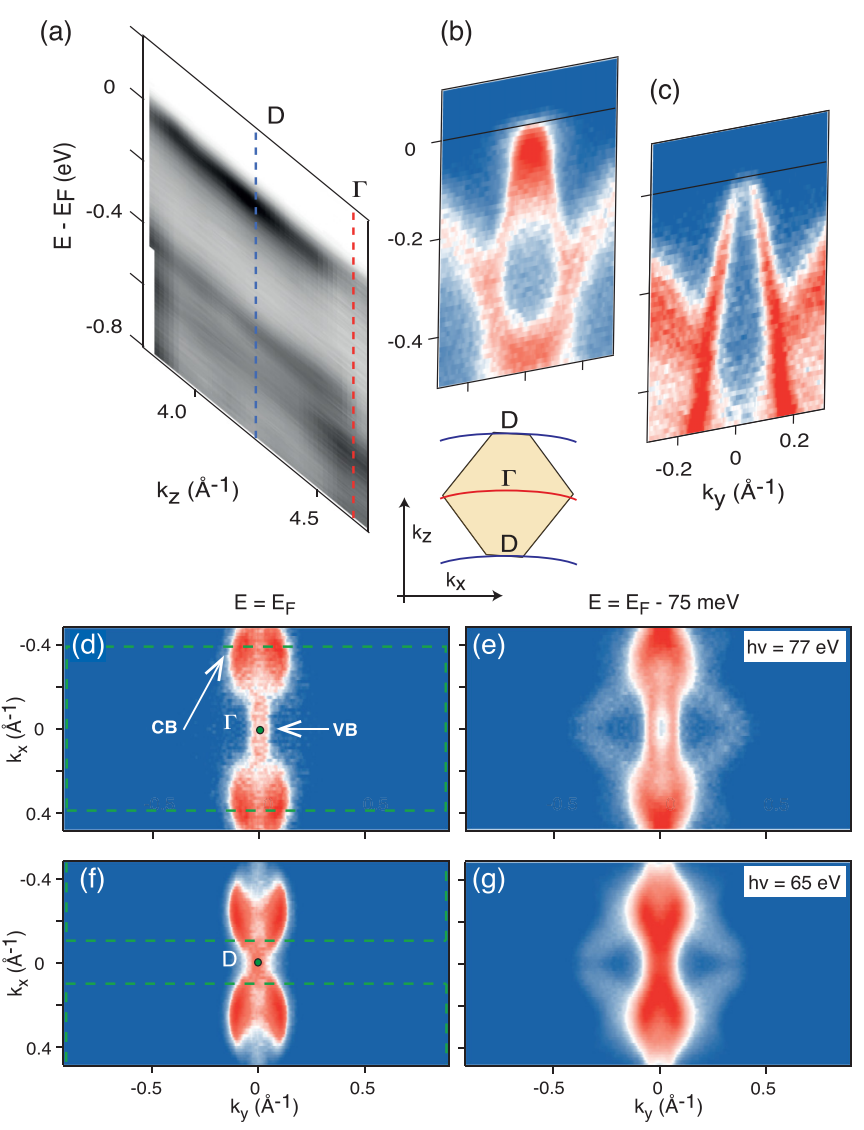

FIG. 3. (a) Band dispersion along $k_{z}$ as obtained by photon energy dependent ARPES, for a value of inner potential $V_{0}=10 \mathrm{eV}$. The $k_{z}$ direction forms a small angle with respect to the bulk $\Gamma D$ direction. Intensity disperses below $E_{\mathrm{F}}$ when moving from the $\Gamma$ point (red line) of the sixth BZ towards $D$ (blue line), which is accessible for a photon energies closer to $65 \mathrm{eV}$. (b) and (c) Band dispersions measured along $k_{y}$ for photon energies equal to $65 \mathrm{eV}$ (b) and $77 \mathrm{eV}$ (c). In the former we notice the appearance of extra intensity near $D$ due to the presence of CB. (d) and (e) Constant energy maps at $E_{\mathrm{F}}$ and $75 \mathrm{meV}$ below, measured at $77 \mathrm{eV}$. This fixed photon energy correspond to a curved surface in the reciprocal space passing through the bulk $\Gamma$ point. (f) and (g) The same as in (d) and (e), but for $65 \mathrm{eV}$, corresponding to a surface passing near $D$. All the constant energy maps have been symmetrized on the plane $k_{x}=0 \AA^{-1}$.

the experiment, we project the Fermi surface on few selected planes for $k_{z}=0$ (b), $k_{z}=0.2$ (c), and $k_{z}=0.5$ (d), in units of the periodicity along the out-of-plane direction. We find that $\mathrm{CB}$ is made by two sheets, and not just a single ellipsoid. Experimentally we cannot resolve the splitting due to the limited momentum resolution achieved for the investigated photon energy range, but a recent laser based ARPES study confirms the topology of CB [38]. Figure 4(c) shows that VB forms additional sheets which are accessible only for a small range of $k_{z}$ values and that are not observed in our experiment, probably due to the limited $k_{z}$ resolution. This shows how theory can assist ARPES in providing a complete picture of the 3D Fermi surface.

As a matter of fact, our experiment overestimates the electron density and it underestimates the hole density. On the (a)
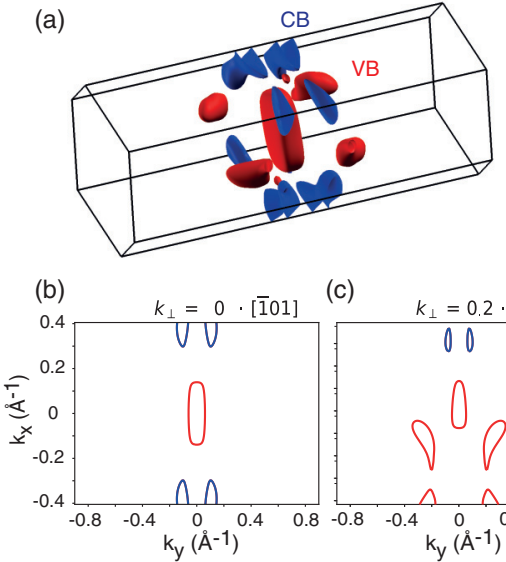

(c)

$k_{\perp}=0.2 \cdot[\overline{1} 01]$

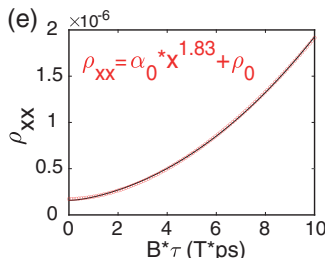

(d)
FIG. 4. (a) Calculated 3D Fermi surface of $\mathrm{TaSe}_{3}$, blue and red colors distinguish the electron and hole contributions. (b)-(d) Projections of the Fermi surface onto three planes for $k_{z}=0$ (b), $k_{z}=0.2$ (c), and $k_{z}=0.5$ (d), in units of the periodicity along the out-of-plane direction. (e) Red circles show the simulation of longitudinal MR based on the calculated Fermi surface as a function of $B \cdot \tau$, the product between the magnetic field and the characteristic relaxation time. A black line shows the best fit, with a characteristic exponent of 1.83 that well captures the quadratic behavior of the MR.

basis of the calculated Fermi surface, we carried out advanced simulations of the transport properties by using Boltzmann theory within a semiclassical model and relaxation time approximation. Figure 4(e) shows the simulated longitudinal MR as a function of $B \cdot \tau$, the product between the magnetic field and the characteristic relaxation time. Theory well reproduces the quadratic nonsaturating MR, and this signifies how important the role played by electron-hole compensation and Fermi surface topology is in $\mathrm{TaSe}_{3}$.

We finally remark that the experimental difference in the areas of $\mathrm{CB}$ and $\mathrm{VB}$ might reflect also the shift of the $E_{\mathrm{F}}$, which we already noticed in Fig. 2. Although we attribute this $n$ doping to defects and Se vacancies created at the surface in the cleavage process, we cannot fully rule out that small doping might be present also in the bulk, partially disrupting the perfect electron-hole compensation. Additional ingredients might be taken into account to explain the measured MR. Recently, Tafti and co-workers have discussed the role of large $p-d$ orbital mixing, whose hybridization can be modified by the action of an external $B$ field [18]. In $\mathrm{TaSe}_{3}$ the $p$ - $d$ orbital hybridization arises from the inversion of the energy ordering between the Ta $d$ and the Se $p$ levels, due to crystal field effect in the prismatic cage structure, combined with the action of strong spin-orbit coupling that opens a hybridization gap at the crossing between those levels [30]. Unfortunately this term is not included in our simulations of the magnetotransport and we cannot quantify its role.

\section{Topology and band-gap engineering}

According to the theoretical study of Nie and coauthors [30], $\mathrm{TaSe}_{3}$ should realize a STI, in contrast with our findings that predict a WTI phase, where an additional band inversion occurs. The transition towards the trivial semimetallic phase would require all the band-gap inversions to be removed. The 

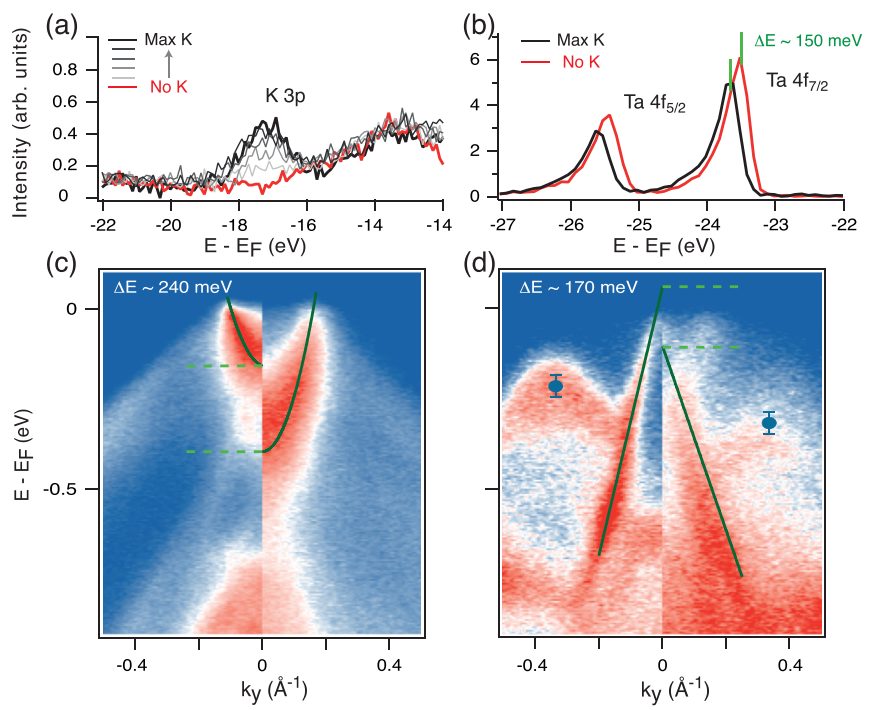

FIG. 5. (a) Evolution of the K $3 p$ core level as a function of doping. (b) Change in the binding energy of the Ta $4 f$ core levels before (red) $\mathrm{K}$ deposition and at maximum coverage (black). (c) Change in band dispersion along $\overline{X M}$, as measured at $65 \mathrm{eV}$, before (left) $\mathrm{K}$ deposition and at maximum coverage (right). The position of $\mathrm{CB}$ is analyzed by fitting MDCs, and the resulting peak positions are well approximated by parabolic bands shown in green. (d) The same as (c) but for VB measured at $77 \mathrm{eV}$. Blue markers indicate the position of the secondary VB maximum as estimated from a fit of the EDC.

stability of the TI phases has been tested by computing the topological invariants for different values of hydrostatic strain, and variations up to $\pm 5 \%$ are not sufficient to bring the system into the trivial phase, in good agreement with the results in the literature [30]. The discrepancy between the computed topological phases likely arises from the fact that our calculations are based on the experimental atomic positions [37], while ionic positions were free to relax in the above-mentioned work [30]. For van der Waals materials, it is well known that this procedure leads to an artificial expansion of the interlayer distances.

Having clear in mind that $\mathrm{TaSe}_{3}$ lies in proximity of a topological phase transition, it is interesting to consider the possibility to manipulate its topological phase. From the computational point of view, pressure and strain are ideal knobs. While the former is difficult to combine with ARPES experiment, there is an increasing number of reports of straindependent ARPES studies [57-59]. Such an approach has been recently exploited to manipulate the topology of $\mathrm{TaSe}_{3}$ [39]. An alternative route is offered by evaporation of alkali metals, which has been recently reported to engineer the band gap in many layered materials. Absorption of alkali metals, in fact, does not induce a rigid band shift, but in transition metal dichalcogenides [60-62], in group III monochalcogenides [63], and in black phosphorus [64], it is responsible for a band-gap renormalization that can even result in the full closure of the gap and a topological phase transition [65]. We have adopted a similar strategy for $\mathrm{TaSe}_{3}$, and the results are summarized in Fig. 5.

Figure 5(a) shows the increase in the $\mathrm{K} 3 p$ core level, which we monitor upon subsequent cycles of evaporations.
Figure 5(b) compares the Ta $4 f$ levels before adsorption (red) and at maximum $\mathrm{K}$ coverage (black); a shift of $150 \pm 50 \mathrm{meV}$ is observed. Figures 5(c) and 5(d) show the effect of $\mathrm{K}$ on $\mathrm{CB}$ and $\mathrm{VB}$, along $\overline{X M}$ and $\overline{\Gamma Y}$, respectively. We directly compare the dispersion before $\mathrm{K}$ evaporation (left) and at maximum coverage (right). The dispersion of $\mathrm{CB}$ and $\mathrm{VB}$ is analyzed quantitatively by fitting MDCs. The peak positions are then approximated by a simple parabolic dispersion (CB) and by a linear dispersion (VB) and the results are shown as green lines. The minimum of $\mathrm{CB}$ is found to shift by $\Delta E_{\mathrm{CB}}=240 \pm 20 \mathrm{meV}$ while $\mathrm{VB}$ experiences a shift of $\Delta E_{\mathrm{VB}}=170 \pm 20 \mathrm{meV}$. A smaller shift is consistently observed also for the secondary maximum of VB. In Fig. 5(d) we indicate with blue circles the positions as extracted from a fit to the EDCs, yielding a shift of $\Delta E_{\mathrm{VB}}=120 \pm 30 \mathrm{meV}$. Therefore, the bottom of $\mathrm{CB}$ is lower in energy more than the maximum of VB. Such an orbital-dependent shift could be used to control the ordering of the energy levels, and the resulting $p-d$ hybridization. One could anticipate devices based on exfoliation of $\mathrm{TaSe}_{3}$, since an even larger renormalization of the band gap should be achievable by external electric fields.

\section{E. Surface states and spin polarization}

The observation of special surface states (SSs) would establish the topological phase of the material. Nie and coworkers proposed that, in $\mathrm{TaSe}_{3}$, the fingerprint of the STI phase is a SS linearly dispersing around the $\bar{X}$ point of the (101) surface. However, our results clarify that the observation of this state is not sufficient to ensure the STI phase alone, since our calculations of Fig. 2(d) shows that a similar state, labeled SS1, is present also in the WTI phase. It is important to recall that WTIs possess special surfaces, dubbed dark surfaces, where no topological SS are present [66]. For $\mathrm{TaSe}_{3}$, the (101) termination is a dark surface, and therefore SS1 must be topologically trivial.

Although we cannot experimentally resolve the dispersion of SS1 from that of CB [Figs. 2(d) and 5(c)], the calculations of Fig. 2(d) predicts the existence of a second surface state, labeled SS2, which exhibits large spin polarization. In order to further discuss this property of $\mathrm{TaSe}_{3}$, we need to introduce a technical aspect, which we have neglected so far: the existence of two different terminations. Figures 6(a) and 6(b) show the dispersion along $\overline{\Gamma Y}$ measured at $77 \mathrm{eV}$ for the two terminations, labeled A and B. We do not observe major differences between the two band structures, except for a large transfer of spectral weight in B from the top of VB to SS2.

The enhanced spectral weight of SS2 allows us to investigate its spin polarization by means of srARPES. Figure 6(c) displays the calculated spin polarization along the same high-symmetry direction, SS2 is spin-split and one of its component is well isolated within the projected band gap below the secondary VB maximum. In the region where its dispersion becomes linear, SS2 hybridizes with VB that inherits a small spin polarization. These calculations support our experimental findings. Figure 6(d) shows the spin polarization, obtained by collecting spin EDCs for the component of the spin vector orthogonal to the wave vector, in the region of band structure highlighted by the green rectangle in Fig. 6(b). 
(a)

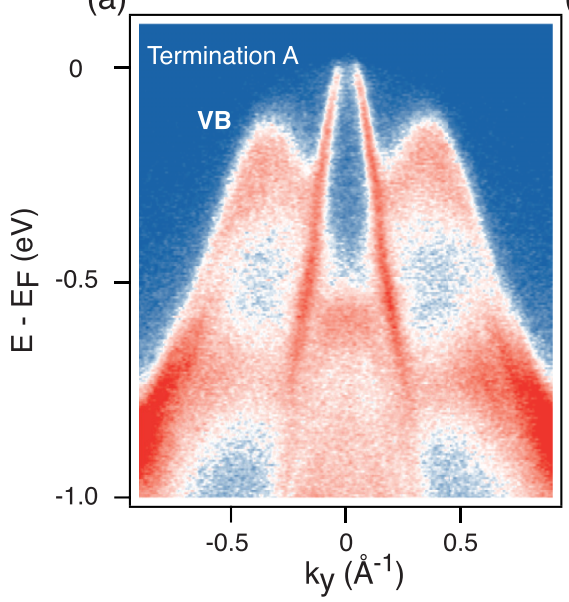

(c)

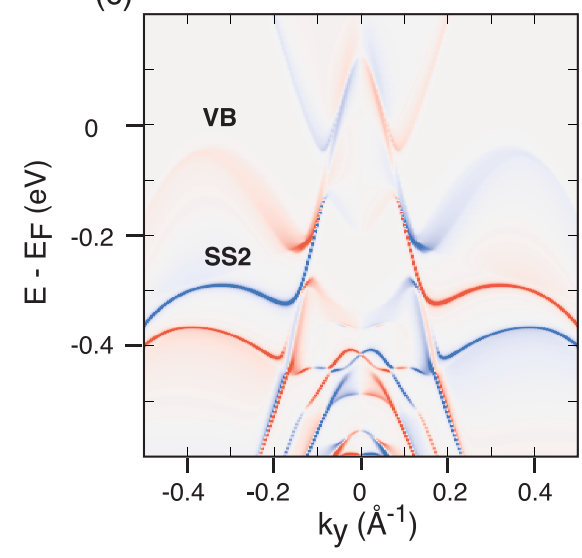

(b)
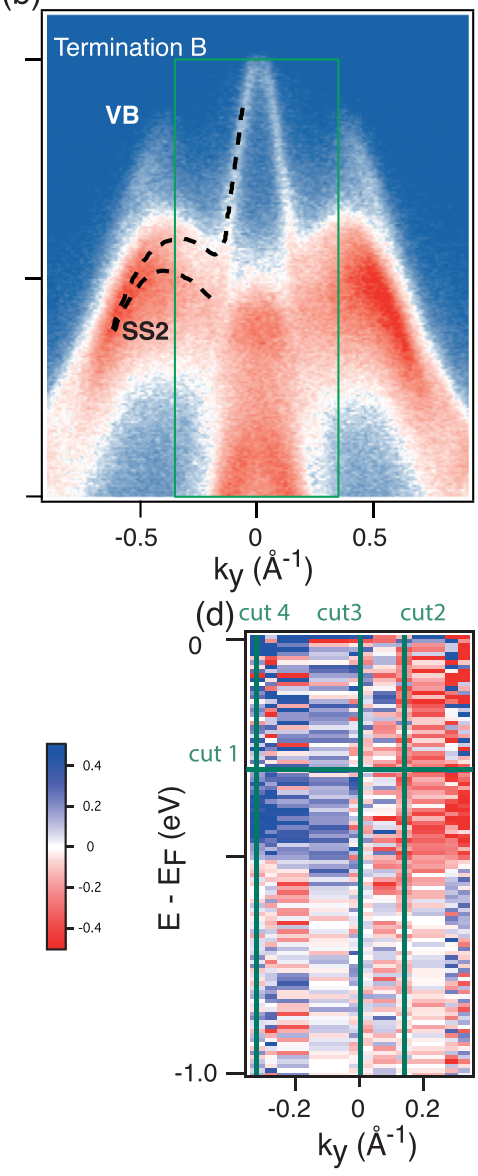

(e)

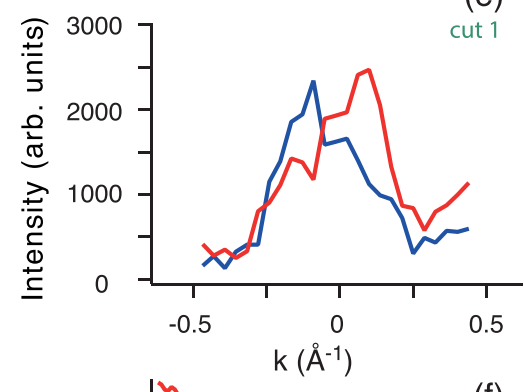

(f)

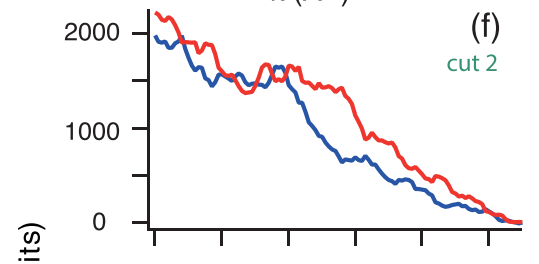

(g)

cut 3

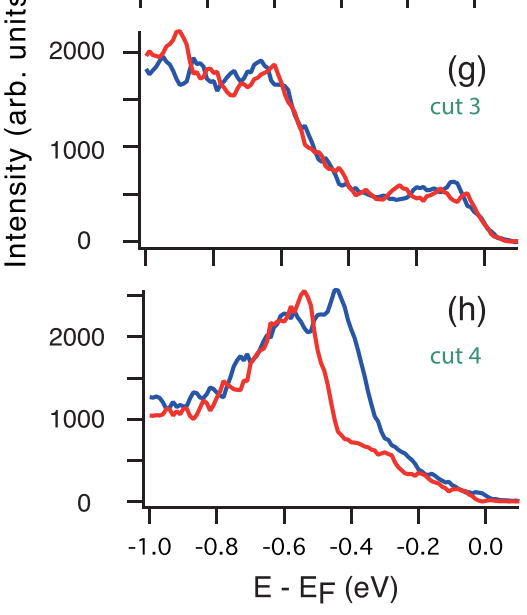

FIG. 6. (a) and (b) Band dispersion along $k_{y}$ measured at $77 \mathrm{eV}$ for the two terminations, labeled A and B. A large transfer of spectral weight between VB and SS is observed in B. (c) Calculated spin polarization along the same direction, for the spin component orthogonal to the wave vector. (d) Measured spin polarization in the area of the band structure highlighted in (b) by a green rectangle. (e)-(h) Spin MDC and spin EDCs taken along selected cuts indicated by numbers in (d).

We resolve a large spin polarization in the region of SS2, and the spin EDCs corresponding to the wave vectors of cuts 2-4 are shown in Figs. 6(f)-6(h). Along the linear dispersion of SS, the spin polarization is better resolved in the spin MDC measured along cut 1 and shown in Fig. 6(e). We can conclude that future srARPES studies might identify the dispersion of the topological SS1, by focusing in particular on the termination B.

\section{CONCLUSIONS}

In summary, we have elucidated the relation between large nonsaturating quadratic MR and Fermi surface topology in $\mathrm{TaSe}_{3}$. We have investigated its band dispersion and the Fermi surface by combining ARPES and ab initio calculations. Simulations based on the calculated Fermi surface suggest that electron-hole compensation is responsible for the observed MR. We experimentally show that the material's band gap can be engineered by deposition of alkali metals, which might be exploited to induce topological phase transition in alter- native to strain. All together our results illustrate that $\mathrm{TaSe}_{3}$ combine nonsaturating MR, topology, nonsymmorphic symmetry, with high mobility and a band velocity as large as that of $\mathrm{Cd}_{3} \mathrm{As}_{2}$ and $\mathrm{ZrTe}_{5}$, all embedded in a material that exhibits also a superconducting transition. $\mathrm{TaSe}_{3}$ represents thereby an ideal platform for realizing exotic quantum phenomena, such as fractional quantum Hall effect, anomalous Hall effect, Majorana fermions, and topological superconductivity.

\section{ACKNOWLEDGMENTS}

We acknowledge illuminating discussions with Marco Grioni. We acknowledge financial support by the Swiss National Science Foundation (SNSF). We acknowledge the support by the NCCR Marvel. All first-principles calculations were performed at the Swiss National Supercomputing Centre (CSCS) under the Project No. s1008. This work has been partly performed in the framework of the nanoscience foundry and fine analysis (NFFA-MUR Italy Progetti Internazionali) facility. We acknowledge financial support by Sino-Swiss Science and Cooperation Programme Grant No. EG 01-122016. 
[1] P. Kapitza, Proc. R. Soc. London Ser. A 119, 358 (1928).

[2] M. N. Baibich, J. M. Broto, A. Fert, F. Nguyen Van Dau, F. Petroff, P. Etienne, G. Creuzet, A. Friederich, and J. Chazelas, Phys. Rev. Lett. 61, 2472 (1988).

[3] J. M. Daughton, J. Magn. Magn. Mater. 192, 334 (1999).

[4] T. Miyazaki and N. Tezuka, J. Magn. Magn. Mater. 139, L231 (1995).

[5] G. A. Prinz, Science 282, 1660 (1998).

[6] A. Fert, Rev. Mod. Phys. 80, 1517 (2008).

[7] M. N. Ali, J. Xiong, S. Flynn, J. Tao, Q. D. Gibson, L. M. Schoop, T. Liang, N. Haldolaarachchige, M. Hirschberger, N. P. Ong et al., Nature (London) 514, 205 (2014).

[8] N. P. Armitage, E. J. Mele, and A. Vishwanath, Rev. Mod. Phys. 90, 015001 (2018).

[9] J. Hu, S.-Y. Xu, N. Ni, and Z. Mao, Annu. Rev. Mater. Res. 49, 207 (2019).

[10] T. Liang, Q. Gibson, M. N. Ali, M. Liu, R. J. Cava, and N. P. Ong, Nat. Mater. 14, 280 (2015).

[11] X. Huang, L. Zhao, Y. Long, P. Wang, D. Chen, Z. Yang, H. Liang, M. Xue, H. Weng, Z. Fang, X. Dai, and G. Chen, Phys. Rev. X 5, 031023 (2015).

[12] A. Collaudin, B. Fauqué, Y. Fuseya, W. Kang, and K. Behnia, Phys. Rev. X 5, 021022 (2015).

[13] K. Wang, D. Graf, L. Li, L. Wang, and C. Petrovic, Sci. Rep. 4, 7328 (2014).

[14] B. Shen, X. Deng, G. Kotliar, and N. Ni, Phys. Rev. B 93, 195119 (2016).

[15] F. F. Tafti, Q. D. Gibson, S. K. Kushwaha, N. Haldolaarachchige, and R. J. Cava, Nat. Phys. 12, 272 (2016).

[16] J. He, C. Zhang, N. J. Ghimire, T. Liang, C. Jia, J. Jiang, S. Tang, S. Chen, Y. He, S.-K. Mo, C. C. Hwang, M. Hashimoto, D. H. Lu, B. Moritz, T. P. Devereaux, Y. L. Chen, J. F. Mitchell, and Z. X. Shen, Phys. Rev. Lett. 117, 267201 (2016).

[17] Z. Yuan, H. Lu, Y. Liu, J. Wang, and S. Jia, Phys. Rev. B 93, 184405 (2016).

[18] F. F. Tafti, Q. Gibson, S. Kushwaha, J. W. Krizan, N. Haldolaarachchige, and R. J. Cava, Proc. Natl. Acad. Sci. USA 113, E3475 (2016).

[19] S. Mangelsen, P. G. Naumov, O. I. Barkalov, S. A. Medvedev, W. Schnelle, M. Bobnar, S. Mankovsky, S. Polesya, C. Näther, H. Ebert, and W. Bensch, Phys. Rev. B 96, 205148 (2017).

[20] I. A. Leahy, Y.-P. Lin, P. E. Siegfried, A. C. Treglia, J. C. W. Song, R. M. Nandkishore, and M. Lee, Proc. Natl. Acad. Sci. USA 115, 10570 (2018).

[21] A. B. Pippard, Magnetoresistance in Metals (Cambridge University Press, Cambridge, 2009).

[22] E. Fawcett and W. A. Reed, Phys. Rev. 131, 2463 (1963).

[23] S. Zhang, Q. Wu, Y. Liu, and O. V. Yazyev, Phys. Rev. B 99, 035142 (2019).

[24] I. Pletikosić, M. N. Ali, A. V. Fedorov, R. J. Cava, and T. Valla, Phys. Rev. Lett. 113, 216601 (2014).

[25] D. Rhodes, S. Das, Q. R. Zhang, B. Zeng, N. R. Pradhan, N. Kikugawa, E. Manousakis, and L. Balicas, Phys. Rev. B 92, 125152 (2015).

[26] C.-L. Wang, Y. Zhang, J.-W. Huang, G.-D. Liu, A.-J. Liang, Y.-X. Zhang, B. Shen, J. Liu, C. Hu, Y. Ding et al., Chin. Phys. Lett. 34, 097305 (2017).
[27] S. Thirupathaiah, R. Jha, B. Pal, J. S. Matias, P. K. Das, P. K. Sivakumar, I. Vobornik, N. C. Plumb, M. Shi, R. A. Ribeiro, and D. D. Sarma, Phys. Rev. B 95, 241105(R) (2017).

[28] Y. Wu, N. H. Jo, M. Ochi, L. Huang, D. Mou, S. L. Bud'ko, P. C. Canfield, N. Trivedi, R. Arita, and A. Kaminski, Phys. Rev. Lett. 115, 166602 (2015).

[29] J. Jiang, F. Tang, X. C. Pan, H. M. Liu, X. H. Niu, Y. X. Wang, D. F. Xu, H. F. Yang, B. P. Xie, F. Q. Song, P. Dudin, T. K. Kim, M. Hoesch, P. K. Das, I. Vobornik, X. G. Wan, and D. L. Feng, Phys. Rev. Lett. 115, 166601 (2015).

[30] S. Nie, L. Xing, R. Jin, W. Xie, Z. Wang, and F. B. Prinz, Phys. Rev. B 98, 125143 (2018).

[31] L. Fu, C. L. Kane, and E. J. Mele, Phys. Rev. Lett. 98, 106803 (2007).

[32] M. Grioni, S. Pons, and E. Frantzeskakis, J. Phys.: Condens. Matter 21, 023201 (2009).

[33] P. Monceau, Adv. Phys. 61, 325 (2012).

[34] E. Canadell, I. E.-I. Rachidi, J. P. Pouget, P. Gressier, A. Meerschaut, J. Rouxel, D. Jung, M. Evain, and M.-H. Whangbo, Inorg. Chem. 29, 1401 (1990).

[35] A. Perucchi, C. Søndergaard, S. Mitrovic, M. Grioni, N. Barisic, H. Berger, L. Forró, and A. L. Degiorgi, Eur. Phys. J. B 39, 433 (2004).

[36] P. Haen, F. Lapierre, P. Monceau, M. Nunez-Reguiero, and J. Richard, Solid State Commun. 26, 725 (1978).

[37] E. Byerkellund and A. Kjekshus, Acta Chem. Scand. 19, 701 (1965).

[38] C. Chen, A. Liang, S. Liu, S. Nie, J. Huang, M. Wang, Y. Li, D. Pei, H. Yang, H. Zheng et al., Matter 3, 2055 (2020).

[39] C. Lin, M. Ochi, R. Noguchi, K. Kuroda, M. Sakoda, A. Nomura, M. Tsubota, P. Zhang, C. Bareille, K. Kurokawa, Y. Arai, K. Kawaguchi, H. Tanaka, K. Yaji, A. Harasawa, M. Hashimoto, D. Lu, S. Shin, R. Arita, S. Tanda, and T. Kondo, Nat. Mater. 20, 1093 (2021).

[40] E. N. Dolgov, Yu. I. Latyshev, and V. E. Minakova, Sov. Phys. Solid State 26, 2089 (1984).

[41] G. Manzoni, L. Gragnaniello, G. Autès, T. Kuhn, A. Sterzi, F. Cilento, M. Zacchigna, V. Enenkel, I. Vobornik, L. Barba, F. Bisti, P. Bugnon, A. Magrez, V. N. Strocov, H. Berger, O. V. Yazyev, M. Fonin, F. Parmigiani, and A. Crepaldi, Phys. Rev. Lett. 117, 237601 (2016).

[42] C. Bigi et al., J. Synchrotron Rad. 24, 750 (2017).

[43] P. Giannozzi, S. Baroni, N. Bonini, M. Calandra, R. Car, C. Cavazzoni, D. Ceresoli, G. L. Chiarotti, M. Cococcioni, I. Dabo et al., J. Phys.: Condens. Matter 21, 395502 (2009).

[44] A. Umerski, Phys. Rev. B 55, 5266 (1997).

[45] A. A. Mostofi, J. R. Yates, G. Pizzi, Y.-S. Lee, I. Souza, D. Vanderbilt, and N. Marzari, Comput. Phys. Commun. 185, 2309 (2014).

[46] Q. Wu, S. Zhang, H.-F. Song, M. Troyer, and A. A. Soluyanov, Comput. Phys. Commun. 224, 405 (2018).

[47] M. Novak, S. N. Zhang, F. Orbanić, N. Biliškov, G. Eguchi, S. Paschen, A. Kimura, X. X. Wang, T. Osada, K. Uchida, M. Sato, Q. S. Wu, O. V. Yazyev, and I. Kokanovic, Phys. Rev. B 100, 085137 (2019).

[48] Q. Chen, Z. Lou, S. N. Zhang, B. Xu, Y. Zhou, H. Chen, S. Chen, J. Du, H. Wang, J. Yang, Q. S. Wu, O. V. Yazyev, and M. Fang, Phys. Rev. B 102, 165133 (2020). 
[49] Y. Zhou, Z. Lou, S. N. Zhang, H. Chen, Q. Chen, B. Xu, J. Du, J. Yang, H. Wang, C. Xi, L. Pi, Q. S. Wu, O. V. Yazyev, and M. Fang, Phys. Rev. B 102, 115145 (2020).

[50] N. W. Ashcroft and N. D. Mermin, Solid State Physics (Harcourt, Orlando, FL, 1976).

[51] Y. Liu, H.-J. Zhang, and Y. Yao, Phys. Rev. B 79, 245123 (2009)

[52] S. Borisenko, Q. Gibson, D. Evtushinsky, V. Zabolotnyy, B. Büchner, and R. J. Cava, Phys. Rev. Lett. 113, 027603 (2014).

[53] S. Roth, H. Lee, A. Sterzi, M. Zacchigna, A. Politano, R. Sankar, F. C. Chou, G. Di Santo, L. Petaccia, O. V. Yazyev, and A. Crepaldi, Phys. Rev. B 97, 165439 (2018).

[54] Q. Li, D. E. Kharzeev, C. Zhang, Y. Huang, I. Pletikosić, A. V. Fedorov, R. D. Zhong, J. A. Schneeloch, G. D. Gu, and T. Valla, Nat. Phys. 12, 550 (2016).

[55] G. Manzoni, A. Sterzi, A. Crepaldi, M. Diego, F. Cilento, M. Zacchigna, P. Bugnon, H. Berger, A. Magrez, M. Grioni, and F. Parmigiani, Phys. Rev. Lett. 115, 207402 (2015).

[56] A. Bansil, M. Lindroos, S. Sahrakorpi, and R. S. Markiewicz, Phys. Rev. B 71, 012503 (2005).

[57] S. Riccò, M. Kim, A. Tamai, S. M. Walker, F. Bruno, I. Cucchi, E. Cappelli, C. Besnard, T. Kim, P. Dudin et al., Nat. Commun. 9, 4535 (2018).

[58] H. Pfau, S. D. Chen, M. Yi, M. Hashimoto, C. R. Rotundu, J. C. Palmstrom, T. Chen, P.-C. Dai, J. Straquadine, A. Hristov, R. J.
Birgeneau, I. R. Fisher, D. Lu, and Z. X. Shen, Phys. Rev. Lett. 123, 066402 (2019).

[59] V. Sunko, E. A. Morales, I. Marković, M. E. Barber, D. Milosavljević, F. Mazzola, D. A. Sokolov, N. Kikugawa, C. Cacho, P. Dudin et al., npj Quantum Mater. 4, 46 (2019).

[60] M. Kang, B. Kim, S. H. Ryu, S. W. Jung, J. Kim, L. Moreschini, C. Jozwiak, E. Rotenberg, A. Bostwick, and K. S. Kim, Nano Lett. 17, 1610 (2017).

[61] Y. Nakata, K. Sugawara, A. Chainani, K. Yamauchi, K. Nakayama, S. Souma, P.-Y. Chuang, C.-M. Cheng, T. Oguchi, K. Ueno, T. Takahashi, and T. Sato, Phys. Rev. Materials 3, 071001(R) (2019).

[62] A. Rossi, G. Resta, S. H. Lee, R. D. Redwing, C. Jozwiak, A. Bostwick, E. Rotenberg, S. Y. Savrasov, and I. M. Vishik, Phys. Rev. B 102, 121110 (2020).

[63] Z. Zhang, Z. Chen, M. Bouaziz, C. Giorgetti, H. Yi, J. Avila, B. Tian, A. Shukla, L. Perfetti, D. Fan et al., ACS Nano 13, 13486 (2019).

[64] J. Kim, S. S. Baik, S. H. Ryu, Y. Sohn, S. Park, B.-G. Park, J. Denlinger, Y. Yi, H. J. Choi, and K. S. Kim, Science 349, 723 (2015).

[65] J. Kim, S. S. Baik, S. W. Jung, Y. Sohn, S. H. Ryu, H. J. Choi, B.-J. Yang, and K. S. Kim, Phys. Rev. Lett. 119, 226801 (2017).

[66] Y. Yoshimura, A. Matsumoto, Y. Takane, and K.-I. Imura, Phys. Rev. B 88, 045408 (2013). 